\title{
Could Gravitons from a Prior Universe Survive a (LQG Inspired) “Quantum Bounce” to Re-Appear in Our Present Universe?
}

\author{
Andrew Walcott Beckwith \\ Physics Department, Chongqing University, College of Physics, Chongqing University Huxi Campus, Chongqing, China \\ Email: Rwill9955b@gmail.com, abeckwith@uh.edu
}

How to cite this paper: Beckwith, A.W. (2017) Could Gravitons from a Prior Universe Survive a (LQG Inspired) "Quantum Bounce" to Re-Appear in Our Present Universe? Journal of High Energy Physics, Gravitation and Cosmology, 3, 624-634. https://doi.org/10.4236/jhepgc.2017.34047

Received: May 27, 2017

Accepted: September 27, 2017

Published: September 30, 2017

Copyright $\odot 2017$ by author and Scientific Research Publishing Inc. This work is licensed under the Creative Commons Attribution International License (CC BY 4.0).

http://creativecommons.org/licenses/by/4.0/

\begin{abstract}
We ask the question if a formula for entropy, as given by $S \equiv E / T$ with a usual value ascribed of initial entropy $S \sim 10^{5}$ of the onset of inflation can allow an order of magnitude resolution of the question of if there could be a survival of a graviton from a prior to the present universe, using typical Planckian peak temperature values of $T \sim 10^{19} \mathrm{GeV}$. We obtain values consistent with up to $10^{38}$ gravitons contributing to an energy value of $E \sim 10^{24} \mathrm{GeV}$ if we assume a relic energy contribution based upon each graviton initially exhibiting a frequency spike of $10^{10} \mathrm{~Hz}$. The value of $E \sim 10^{24} \mathrm{GeV}$ is picked from looking at the aftermath of what happens if there exists a quantum bounce with a peak density value of $\rho_{\text {maximum }} \sim 2.07 \cdot \rho_{\text {planck }}[1]$ in a regime of LQG bounce regime radii of the order of magnitude of $\lambda \sim 10^{-35}$ meters. The author, in making estimates specifically avoids using $S \equiv[E-\mu N] / T$, by setting the chemical potential $\mu \equiv 0$ for ultra high temperatures for reasons which will be brought up in the conclusion.
\end{abstract}

\section{Keywords}

Gravitons, Prior Universe, Quantum Bounce

\section{Introduction}

Recently, a big bounce has been proposed ${ }^{1}$ as an alternative to singularity conditions that Hawking's, Ellis [2], and others use. The $1^{\text {st }}$ problem is that there appears to exist no fundamental argument presented in either traditional Friedman

${ }^{1}$ Papers on LCQ at the 12th Marcell Grossman Meeting in 2009 (http://www.icra.it/MG/mg12/en/) 
metric GR or LQG for preservation of the same value for Planck's constant or the fine structure constant from prior universes (before ours) and the present universe. Ashtekar [3], in conversations with the author in the inaugural opening of the Penn State gravity center (2007) told the author that the universe preserves most of its "memory" in cosmological cycles, but the proof of this assertion does not show up in Rovelli's [4] reference on Quantum Gravity. The driving force for this present investigation is due to a conversation the author had with Steinhardt and 'tHooft at the meeting "Fundamental Frontiers of Physics" in a parallel session about LQG, and new developments in it.

\section{What Are Necessary First Principles to Consider in Graviton/GW Detection?}

Modeling how much information may be carried by an individual graviton can be achieved by measuring the graviton via instrumentation. Normalized energy density of gravitational waves, as given by Maggiore [5] is

$$
\Omega_{g w} \equiv \frac{\rho_{g w}}{\rho_{c}} \equiv \int_{v=0}^{v=\infty} \mathrm{d}(\log v) \cdot \Omega_{g w}(v) \Rightarrow h_{0}^{2} \Omega_{g w}(v) \cong 3.6 \cdot\left[\frac{n_{v}}{10^{37}}\right] \cdot\left(\frac{v}{1 \mathrm{kHz}}\right)^{4}
$$

where $n_{v}$ is a frequency-based count of gravitons per unit cell of phase space?

Is Equation (1.1) above fundamental physics? And what is the significance of the $n_{v}$ and $v$ terms with regards to if gravitons could have been cycled from a prior to the present universe? The rest of the document will attempt to answer the question of what ultra high frequency inputs into the $n_{v}$ as well as $v$ term are relevant to, assuming that the quantum bounce model of a "recycled" universe is in part, correct.

\subsection{Estimating the Size of Contribution to Energy in $S \equiv E / T$, Assuming a Frequency $v \sim 10^{10}$ Hertz for Relic Gravitons, If the Standard Chemical Potential Is Effectively $\mu=0$ at the Onset of Creation}

As suggested earlier by Beckwith [6], gravitons may have contributed to the re-acceleration of the universe one billion years ago. When q becomes negative, the rate of acceleration of the universe is actually increasing, rather than slowing down [6] [7]. The suggestion Beckwith made for implementing re acceleration involves correct use of the de celebration parameter, and also looking at the behavior of gravitons. The use of Equation (1.2) below to have re acceleration in the application Beckwith made is dependent upon "heavy gravity" and the rest mass of gravitons in four dimensions having a small mass term.

$$
q=-\frac{\ddot{a} a}{\dot{a}^{2}}
$$

We wish next to consider what happens not a billion years ago, but at the onset of creation itself. If a correct understanding of initial graviton conditions is presented, it may add more credence to the idea of a small graviton mass, in a 
rest frame, which may give backing, in part, to Beckwith's use of Equation (1.2) for re acceleration of the universe, in a manner usually associated with Dark Energy. Here, we are making use of refining the following estimates. In what follows, we will have even stricter bounds upon the energy value (as well as the mass) of the graviton based upon the geometry of the quantum bounce, with a radii of the quantum bounce on the order of $l_{\text {Planck }} \sim 10^{-35}$ meters [6] [8].

$$
\begin{aligned}
& \left.m_{\text {graviton }}\right|_{\text {RELATIVISTIC }}<4.4 \times 10^{-22} h^{-1} \mathrm{eV} / \mathrm{c}^{2} \\
& \Leftrightarrow \lambda_{\text {graviton }} \equiv \frac{\hbar}{m_{\text {graviton }} \cdot c}<2.8 \times 10^{-8} \text { meters }
\end{aligned}
$$

For looking at the onset of creation, with a bounce; if we look at $\rho_{\max } \propto 2.07 \cdot \rho_{\text {planck }}$ for the quantum bounce with a value put in for when $\rho_{\text {planck }} \approx 5.1 \times 10^{99}$ grams $/$ meter $^{3}$, where

$$
E_{\text {eff }} \propto 2.07 \cdot l_{\text {Planck }}^{3} \cdot \rho_{\text {planck }} \sim 5 \times 10^{24} \mathrm{GeV}
$$

Then, taking note of this, one is obtaining having a scaled entropy of $S \equiv E / T \sim 10^{5}$ when one has an initial Planck temperature $T \approx T_{\text {Planck }} \sim 10^{19} \mathrm{GeV}$. One needs, then to consider, if the energy per given graviton is, if a frequency $v \propto 10^{10} \mathrm{~Hz}$ and $E_{\text {graviton-effective }} \propto 2 \cdot h v \approx 5 \times 10^{-5} \mathrm{eV}$, then

$$
S \equiv E_{\text {eff }} / T \sim\left[10^{38} \times E_{\text {graviton-effective }}\left(v \approx 10^{10} \mathrm{~Hz}\right)\right] /\left[T \sim 10^{19} \mathrm{GeV}\right] \approx 10^{5}
$$

Having said that, the $E_{\text {graviton-effective }} \propto 2 \cdot h v \approx 5 \times 10^{-5} \mathrm{eV}$ is $10^{22}$ greater than the rest mass energy of a graviton if $E \sim m_{\text {graviton }}$ [red-shift $\left.\sim 0.55\right] \sim\left(10^{-27} \mathrm{eV}\right)$ grams is taken when applied to Equation (1.2) above.

\subsection{Making Sense of the Factor of $10^{38}$ in Equation (1.5). i.e. How to Reconcile Equation (1.5) with $S \sim n$ Used by Y. Jack Ng for DM Particles in His Entropy/Particle Counting Algorithm?}

Note that J. Y. Ng uses the following. [9] i.e. for DM, $S \sim n$, but this is for DM particles, presumably of the order of mass of a WIMP, i.e.

$m_{\text {WIMP }} \approx 100 \cdot \mathrm{GeV} \sim 10^{11}$ electron volts , as opposed to a relic graviton

Mass-energy relationship:

$$
m_{\text {graviton }}\left(\text { energy }-v \approx 10^{10} \mathrm{~Hz}\right) \approx\left[100 \cdot \mathrm{GeV} \sim 10^{11} \mathrm{eV}-\mathrm{WIMP}\right] \times 10^{-16} \sim 10^{-5} \mathrm{eV}
$$

If one drops the effective energy contribution to $v \approx 10^{0} \sim 1 \mathrm{~Hz}$, as has been suggested, then the relic graviton mass-energy relationship is:

$$
m_{\text {graviton }}\left(\text { energy }-v \approx 10^{0} \mathrm{~Hz}\right) \approx\left[100 \cdot \mathrm{GeV} \sim 10^{11} \mathrm{eV}-\text { WIMP }\right] \times 10^{-26} \sim 10^{-15} \mathrm{eV}
$$

Finally, if one is looking at the mass of a graviton a billion years ago, with

$$
m_{\text {graviton }}(\text { red-shift-value } \sim 0.55) \approx\left[100 \cdot \mathrm{GeV} \sim 10^{11} \mathrm{eV}-\mathrm{WIMP}\right] \times 10^{-38} \sim 10^{-27} \mathrm{eV}
$$

i.e., if one is looking at the mass of a graviton, in terms of its possible value as of a billion years ago, one gets the factor of needing to multiply by $10^{38}$ in order to 
obtain WIMP level energy-mass values, congruent with Y. Jack Ng's $S \sim N$ counting algorithm, i.e., the equivalence relationship for entropy and "particle count" may work out well for the WIMP sized DM candidates, and may break down for the graviton mass-energy problem.

\subsection{The Electro Weak Generation Regime of Space Time for Entropy and Early Universe Graviton Production before Eletro Weak Transitions}

A typical value and relationship between an inflation potential $V[\phi]$, and a Hubble parameter value, $\mathrm{H}$ is

$$
H^{2} \sim V[\phi] / m_{\text {Planck }}^{2}
$$

Also, if we look at the temperature $T^{*}$ occurring about the time of the Electro weak transition, if $T \leq T^{*}$ when $T^{*}=T_{c}$ was a critical value, (of which we can write $v\left(T_{c}\right) / T_{c}>1$, where $v\left(T_{c}\right)$ denotes the Higgs vacuum expectation value at the critical temperature $T_{c}$, i.e. $v\left(T_{c}\right) / T_{c}>1$ according to C. Balazc et al. (2005) [10] and denotes that the electro weak transition was a "strongly first order phase transition") then one can write, by conventional theory that

$$
H \sim 1.66 \cdot\left[\sqrt{\tilde{g}_{*}}\right] \cdot\left[T^{2} / m_{\text {Planck }}^{2}\right]
$$

Here, the factor put in, of $\tilde{g}_{*}$ is the number of degrees of freedom. Kolb and Turner [11] put a ceiling of about $\tilde{g}_{*} \approx 100-120$ in the early universe as of about the electro weak transition. If, however, $\tilde{g}_{*} \sim 1000$ or higher for earlier than that, i.e. up to the onset of inflation for temperatures up to $T \approx T_{\text {Planck }} \sim 10^{19} \mathrm{GeV}$, it may be a way to write, if we also state that $V[\phi] \approx E_{\text {net }}$ that if

$$
S \sim 3 \frac{m_{\text {Plank }}^{2}\left[H=1.66 \cdot \sqrt{\tilde{g}_{*}} \cdot T^{2} / m_{\text {planck }}\right]^{2}}{T} \sim 3 \cdot\left[1.66 \cdot \sqrt{\tilde{g}_{*}}\right]^{2} T^{3}
$$

Should the degrees of freedom hold, for temperatures much greater than $T^{*}$, and with $\tilde{g}_{*} \approx 1000$ at the onset of inflation, for temperatures, rising up to, say $T-10^{19} \mathrm{GeV}$, from initially a very low level, pre inflation, then this may be enough to explain how and why certain particle may arise in a nucleated state, without necessarily being transferred from a prior to a present universe.

I.e. the suggestion being presented is that a more standard thermodynamic dependence of entropy upon temperature, i.e. $S \propto T^{3}$ for values of degrees of freedom may be envisioned if one has $S \propto T^{3}$ when $\tilde{g}_{*} \approx 1000$ or even higher even if $T \sim 10^{19} \mathrm{GeV} \gg T^{*}$ is envisioned, in place of $S \neq T^{3}$ if $T \sim 10^{19} \mathrm{GeV} \gg T^{*}$, and assuming that $\tilde{g}_{*} \neq 1000$, i.e. that an upper limit of $\tilde{g}_{*} \approx 100-110$ in degrees of freedom is all that is permitted.

Furthermore, if one assumes that $S \propto T^{3}$ [11] when $\tilde{g}_{*} \approx 1000$ or even higher even if $T \sim 10^{19} \mathrm{GeV} \gg T^{*}$, then there is the possibility that $S \propto T^{3}$ when $\tilde{g}_{*} \approx 1000$ could also hold, if there was in pre inflationary states very LOW initial temperatures, which rapidly built up in an interval of time, as could 
be given by $0<t<t_{\text {Planck }} \sim 10^{-44}$ seconds which has the following "vacuum nucleation" interpretation which will be given below, as exemplified by the example of a harmonic system having, in a time interval $0<t<\breve{T}$ an infusion of energy, into what is otherwise a typical harmonic oscillatory system. Observe the following argument as given by V. F. Mukhanov, and Swinitzki [12] as to additional particles being "created" due to an infusion of energy in an oscillator, obeying the following equations of motion

$$
\begin{array}{ll}
\ddot{q}(t)+\omega_{0}^{2} q(t)=0, & \text { for } t<0 \text { and } t>\breve{T} ; \\
\ddot{q}(t)-\Omega_{0}^{2} q(t)=0, & \text { for } 0<t<\breve{T}
\end{array}
$$

Given $\Omega_{0} \breve{T} \gg 1$, with a starting solution of $q(t) \equiv q_{1} \sin \left(\omega_{0} t\right)$ if $t<0$, Mukhanov state that for $t>\breve{T}$;

$$
q_{2} \approx \frac{1}{2} \sqrt{1+\frac{\omega_{0}^{2}}{\Omega_{0}^{2}}} \cdot \exp \left[\Omega_{0} \breve{T}\right]
$$

The Mukhanov et al. argument leads to an exercise which Mukhanov et.al. [12] claims is solutions to the exercise yields an increase in number count, as can be given by first setting the oscillator in the ground state with $q_{1}=\omega_{0}^{-1 / 2}$, with the number of particles linked to amplitude by $n=[1 / 2] \cdot\left(q_{0}^{2} \omega_{0}-1\right)$, leading to

$$
n=[1 / 2] \cdot\left(1+\left[\omega_{0}^{2} / \Omega_{0}^{2}\right]\right) \cdot \sinh ^{2}\left[\Omega_{0} \breve{T}\right]
$$

I.e. for non zero $\left[\Omega_{0} \breve{T}\right]$, Equation (1.14) leads to exponential expansion of the numerical state. For sufficiently large $\left[\Omega_{0} \breve{T}\right]$, Equation (1.12) and Equation (1.14) are equivalent to placing of energy into a system, leading to vacuum nucleation. A further step in this direction is given by Mukhanov [12] on page 82 of his book leading to a Bogoluybov particle number density of becoming exponentially large

$$
n \sim \sinh ^{2}\left[m_{0} \eta_{1}\right]
$$

Equation (1.12) to Equation (1.14) are, for sufficiently large $\left[\Omega_{0} \breve{T}\right]$ a way to quantify what happens if initial thermal energy are placed in a harmonic system, leading to vacuum particle "creation" Equation (1.15) is the formal Bogolyubov coefficient limit of particle creation. Note that $\ddot{q}(t)-\Omega_{0}^{2} q(t)=0$, for $0<t<\breve{T}$ corresponds to a thermal flux of energy into a time interval $0<t<\breve{T}$. If $\breve{T} \approx\left[t_{\text {planck }} \propto 10^{-44} \mathrm{sec}\right]$ or some multiple of $t_{\text {planck }}$ and if $\Omega_{0} \propto 10^{10} \mathrm{~Hz}$, then Equation (1.12), and Equation (1.14) plus its generalization as given in Equation (1.15) may be a way to imply either vacuum nucleation, or transport of gravitons from a prior to the present universe. Having said that, the problem of Heavy Gravity raises its ugly head in the following field theory example.

\section{Massive Graviton Field Theories and the Limit$$
m_{\text {graviton }} \rightarrow 0
$$

As given by M. Maggiore [5], and further elaborated upon in [13] the massless equation of the graviton evolution equation takes the form 


$$
\partial_{\mu} \partial^{\sigma} h_{\mu v}=\sqrt{32 \pi G} \cdot\left(T_{\mu v}-\frac{1}{2} \eta_{\mu v} T_{\mu}^{\mu}\right)
$$

When $m_{\text {graviton }} \neq 0$, the above becomes

$$
\left(\partial_{\mu} \partial^{\varpi}-m_{\text {graviton }}\right) \cdot h_{\mu v}=\left[\sqrt{32 \pi G}+\delta^{+}\right] \cdot\left(T_{\mu v}-\frac{1}{3} \eta_{\mu v} T_{\mu}^{\mu}+\frac{\partial_{\mu} \partial_{\nu} T_{\mu}^{\mu}}{3 m_{\text {graviton }}}\right)
$$

The mismatch between these two equations, when $m_{\text {graviton }} \rightarrow 0$ is largely due to $m_{\text {graviton }} h_{\mu}^{\mu} \neq 0$ as $m_{\text {graviton }} \rightarrow 0$, which in turn is due to setting $m_{\text {graviton }} \cdot h_{\mu}^{\mu}=-\left[\sqrt{32 \pi G}+\delta^{+}\right] \cdot T_{\mu}^{\mu}$. The mismatch between these two expressions is one of several reasons for exploring what happens for semi-classical models when $m_{\text {graviton }} \neq 0, m_{\text {graviton }} \sim 10^{-65}$ grams, noting that in QM, a spin 2 only two $m_{\text {graviton }} \neq 0$ has five degrees of freedom, whereas the $m_{\text {graviton }} \rightarrow 0$ gram case has helicity states. Note that string theory treats gravitons as "excitations" of a closed string, as given by Keifer [14], with a term added to a space-time metric, $\bar{g}_{u v}$, such that $g_{u v} \equiv \bar{g}_{u v}+\sqrt{32 \pi G} f_{\mu v}$ with $f_{\mu v}$ a linkage to coherent states of gravitons. This is partly in relation to the Venziano [15] expression of $\Delta x \geq \frac{\hbar}{\Delta p}+\frac{l_{s}^{2}}{\hbar} \Delta p$, where $G \sim g^{2} l_{s}^{2}$. Kieffer [14] gives a correction due to quantum gravity in page 179 of the order of $\left(\frac{m}{M_{\text {Planck }}}\right)^{2}$ If the mass, $m_{\text {graviton }} \sim 10^{-65} \mathrm{~g}$, it will be hard to measure as an individual "particle." But, if $m_{\text {graviton }} \sim 10^{-65} \mathrm{~g}$ exists, as a macro effect one billion years ago, i.e. as a substitute for DE, it also would be potentially relevant toward information exchange between a prior to the present universe, provided that there was no cosmic singularity and that the LQG quantum bounce hypothesis has some validity., Note that the author has been informed by J. Dickau of research by [16] de Rham and Gabadadze which in the authors opinion clears up the problem of ghosts and heavy gravity (massive Gravitons). However, the issue of if a graviton could survive a quantum bounce in LQG [1] stands alone as a problem which the author believes has been removed from being impossible to entertain, to one which cannot be ruled out.

\section{Conclusions}

A way to obtain traces of information exchange, from prior to present universe cycles is finding a linkage between information and entropy. If such a parameterization can be found and analyzed, then Seth Lloyd's [17] shorthand for entropy,

$$
I=S_{\text {total }} / k_{B} \ln 2=[\# \text { operations }]^{3 / 4}=\left[\rho \cdot c^{5} \cdot t^{4} / \hbar\right]^{3 / 4}
$$

could be utilized as a way to represent information which can be transferred from a prior to the present universe. The question to ask, if Equation (1.18) does permit a linkage of gravitons as information carriers, and there can be a linkage of information, in terms of the appearance of gravitons in the time interval of, 
say $0<t<t_{\text {Planck }}$ either by vacuum nucleation of gravitons/information packets along the lines of Equation (1.12) and Equation (1.14) or by reconciling the counting algorithm questions put up in Section 2.2.

\subsection{Further Research Questions for Investigative Inquiry and How to Link Our Inquiry to the Overall Geometry of the Universe}

The problem of reconciling the existence of a graviton mass with quantum mechanics, in spin two particles usually having zero mass appears to be resolvable, and may imply a linkage between DE and DM [6] Furthermore, the radius of the universe problem, as presented by Roos [18], will yield rich applications of the Friedmann equations used in this document, once there are falsifiable experimental criteria for determining both the Hubble Parameter $H=\frac{\dot{a}}{a}$ on the basis of choices of Friedman equations, and $\Omega \equiv \rho(t) / \rho_{\text {critical }}$, using variables chosen and described in this present paper. Both are pertinent to the problem of re-acceleration of the universe parameter set in Equation (1.2), [18]

$$
r_{U} \equiv \frac{1}{H \cdot \sqrt{|\Omega-1|}}
$$

Specifically, the author is convinced that analyzing Equation (1.19) will be tied in, with appropriate analysis of the following Figure 1.

The relation between $\Omega_{g}$ and the spectrum $h\left(v_{g}, \tau\right)$ is often expressed as written by Grishchuk, [20], as

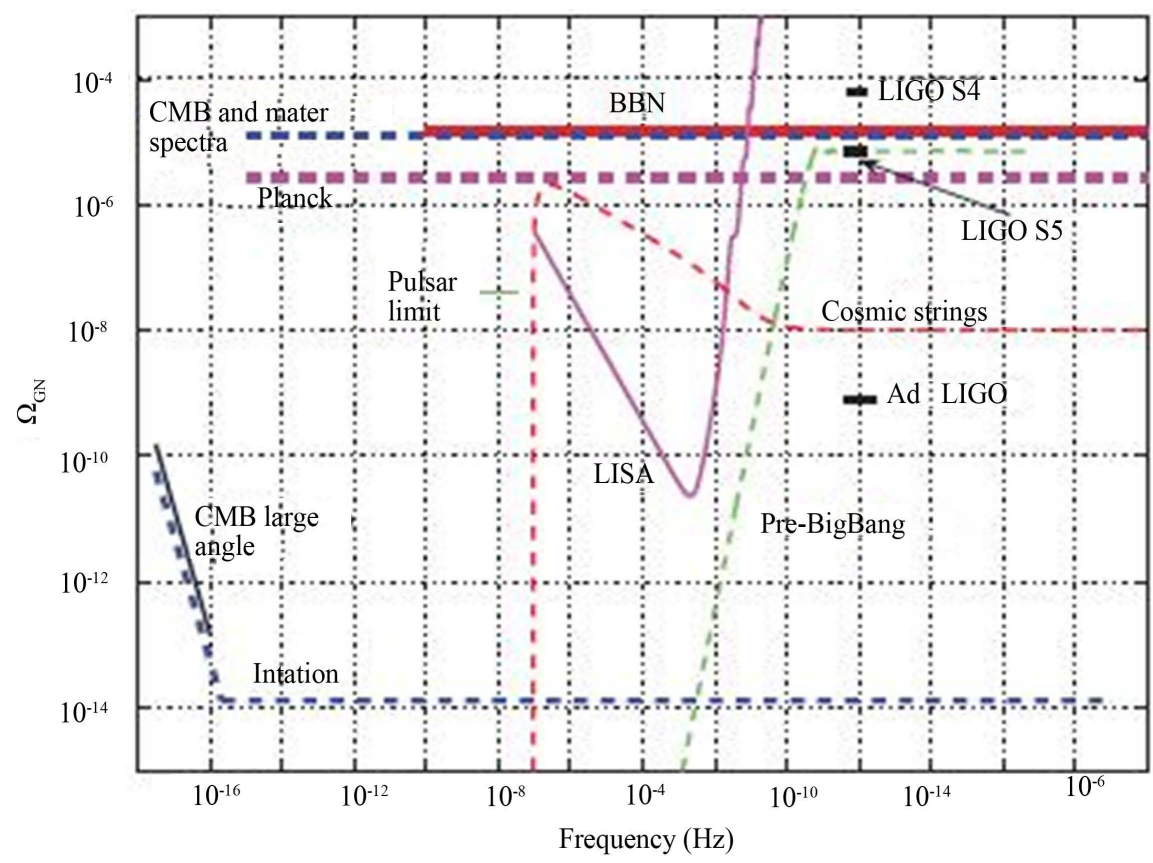

Figure 1. This figure from B. P. Abbott et al. [19] (2009) shows the relation between $\Omega_{g}$ and frequency. 


$$
\Omega_{g} \approx \frac{\pi^{2}}{3}\left(\frac{v}{v_{H}}\right)^{2} h^{2}(v, \tau),
$$

The importance of understanding the radius of the universe question, and making sense of Equation (1.19) lies in reconciling the conflicting estimates put in Section 2.2 above. If one can get an answer to reconciling the estimates put in Section 2.2, one has gone a long way toward answering, or laying the ground work to answering the question as to the classical nature of gravitons, or if they have a semi classical interpretation.

t'Hooft [21], writes, that gravitons can attain mass by spontaneous local symmetry breaking. "The question is whether this can happen in a Lorentz-invariant way". The author submits that when t'Hooft writes that "These arguments should not be regarded as opposed to, but rather complimentary to the AdS/CFT approach to solve QCD using superstring theory [6], where the $3+1$ dimensional theory is mapped onto a 5 dimensional AdS theory. There, the massless graviton in 5 dimensions is mapped onto a massive graviton in 4 dimensions", that one is actually considering, as an example, mapping of higher dimensional contributions of gravitons before the electro weak transition, into the time space $0<t<t_{\text {Planck }}$ which may lead to the construction of newly nucleated graviton states, contributing to, in one sense or another to the different scenarios as given in Section 2.2 above. If a quantum bounce, is the only way, without higher dimensions to answer the issues in Section 2.2, then one has to ask if enough experimental evidence exists to confirm if Equation (1.12) to Equation (1.15) are implying nucleation of gravitons in a relic sense AFTER a LQG big bounce regime of energy transfer, or of an actual transmission of gravitons from a prior universe.

\subsection{Further Inquiry as to If the Chemical Potential, as Given by $\mu \neq 0$ Approaches Zero in the Onset of Inflation/Super Inflation}

Beckwith has very deliberately set $S \equiv[E-\mu N] / T \rightarrow E / T$ with $\mu \neq 0$ approaching zero. Note that L. Glinka [22] in his quantum rendition of a graviton gas has $\mu \neq 0$, and calculates entropy based upon a partition function, explicitly with $\mu \neq 0$ results. Beckwith thinks that Glinka's work is sound, but has decided to set $\mu \neq 0$ to $\mu=0$ for the following reason. The main benefit of chemical potentials is in applications of BBN and/or neutrino physics, i.e. a good example of such is given by Raffelt [23] as of neutrino physics, BBN, and cosmology. At the very onset of inflation which is where the analysis of setting $S \equiv[E-\mu N] / T \rightarrow E / T$ occurs, Beckwith is very deliberately setting initial nucleation at or before the BBN/neutrino physics contributions to cosmology.

If the author, Beckwith, is wrong, he will be quite happy to amend his work along the lines given by L. Glinka's 2007 work [22]. However, in lieu of the absence of either a neutrino physics/BBN contribution, he is attempting to come up with falsifiable experimental results using initially $S \equiv[E-\mu N] / T \rightarrow E / T$ 
and also attempting to make sense of if there is a way to distinguish between the criteria given in Section 2 of this document.

Note that Appendix A below summarizes some of the methods used by the author in terms of counting of gravitons and initial entropy assumed in this document. The reader should also review [23] as well which places the idea of infinite quantum statistics in context.

In addition, in Appendix B, the author gives a summary as to some emerging trends in gravitational wave astronomy which are extremely important. References [24], [25] and [26] are extraordinarily relevant to the ideas brought up and shared here, i.e. that Corda in [26] has outlined protocol as to the emerging issues concerning interferometry and the tests for the nature of gravity is undeniably relevant to our manuscript, and not to mention the speculations on extra dimensions in [27].

\section{Acknowledgements}

This work is supported in part by National Nature Science Foundation of China grant No. 11375279

\section{References}

[1] Malkiewicz, P. and Piechocki, W. (2013) Energy Scale of the Big Bounce. http://arxiv.org/pdf/0903.4352v3

[2] Hawking, S. and Ellis, G.F.R. (1973) The Large Scale Structure of Space-Time. Cambridge University Press, Cambridge. https://doi.org/10.1017/CBO9780511524646

[3] Ashtekar, A. (2007) Private Communications with the Author, at the Penn State Cosmology Center.

[4] Rovelli, C. (2005) Quantum Gravity. Cambridge University Press, Cambridge.

[5] Maggiore, M. (2008) Gravitational Waves, Volume 1: Theory and Experiment. Oxford University Press, Oxford.

[6] Beckwith, A.W. (2010) http://vixra.org/abs/0910.0057

[7] Alves, M., Miranda, O. and de Araujo, J.J. (2009) Can Massive Gravitons Be an Alternative to Dark Energy? http://arxiv.org/abs/0907.5190

[8] Valev, D. (2008) Neutrino and Graviton Rest Mass Estimations by a Phenomenological Approach. Aerospace Research in Bulgaria, 22, 68-82. http://arxiv.org/abs/hep-ph/0507255

[9] Ng, Y. (2008) Spacetime Foam: From Entropy and Holography to Infinite Statistics and Nonlocality. Entropy, 10, 441-461. https://doi.org/10.3390/e10040441

[10] Balazs, C.,. Carena, M, Menon A., Morrissey, D.E. and Wagner, C.E.M. Overview of Electroweak Baryogenesis. 2005 ALCPG \& ILC Workshops-Snowmass, U.S.A. http://www.slac.stanford.edu/econf/C0508141/proc/papers/ALCPG0333.PDF

[11] Kolb, E. and Turner, S. (1994) The Early Universe. Westview Press, Chicago, IL.

[12] Mukhanov, V. and Winitzki, S. (2007) Introduction to Quantum Effects in Gravity. Cambridge University Press, Cambridge. https://doi.org/10.1017/CBO9780511809149

[13] 2011 Gravitational Waves and Experimental Gravity. 
http://moriond.in2p3.fr/Proceedings/2011/Moriond_Grav_2011.pdf

[14] Kiefer, C. (2007) Quantum Gravity. 2nd Edition, Oxford Science Publications, Oxford. https://doi.org/10.1093/acprof:oso/9780199212521.001.0001

[15] Veneziano, G. (1993) Classical and Quantum Gravity from String Theory. In: Bento, M.C., Bertolami, O., Mourao, J.M. and Picken, R.F., Eds., Classical and Quantum Gravity, World Press Scientific, Singapore, 134-180.

[16] De Ramn, C. and Gabadadze, G. (2016) Generalization of the Fierz-Pauli Action. http://arxiv.org/pdf/1007.0443v1

[17] Lloyd, S. (2002) Computational Capacity of the Universe. Physical Review Letters, 88, Article ID: 237901. https://doi.org/10.1103/PhysRevLett.88.237901

[18] Roos, M. (2003) Introduction to Cosmology. 3rd Edition, Wiley Interscience.

[19] Abbott, B.P., et al. (2009) An Upper Limit on the Stochastic Gravitational-Wave background of Cosmological Origin. Nature, 460, 990-993.

https://www.nature.com/nature/journal/v460/n7258/full/nature08278.html https://doi.org/10.1038/nature08278

[20] Grishchuk, L.P. (2001) Relic Gravitational Waves and Their Detection. Lecture Notes in Physics, 562, 167.

[21] Hooft, G. (2008) Unitarity in the Brout-Englert-Higgs Mechanism for Gravity. http://arxiv.org/pdf/0708.3184 v4

[22] Glinka, L. (2007) Quantum Information from Graviton-Matter Gas. Proceedings of the 7 th International Conference "Symmetry in Nonlinear Mathematical Physics", Kyiv, 24-30 June 2007. http://www.emis.de/journals/SIGMA/2007/087/sigma07-087.pdf

[23] Raffelt, G. (2005) Cosmological Neutrinos. Universal Academy Press, Inc. and Yamada Science Foundation. http://wwwth.mppmu.mpg.de/members/raffelt/mypapers/200507.pdf

[24] Abbot, B.P., et al. (2016) Observation of Gravitational Waves from a Binary Black Hole Merger. Physical Review Letters, 116, Article ID: 061102. https://physics.aps.org/featured-article-pdf/10.1103/PhysRevLett.116.061102

[25] Abbot, B.P., et al. (LIGO Scientific Collaboration and Virgo Collaboration) (2016) GW151226: Observation of Gravitational Waves from a 22-Solar-Mass Binary Black Hole Coalescence. Physical Review Letters, 116, Article ID: 241103.

[26] Corda, C. (2009) Interferometric Detection of Gravitational Waves: The Definitive Test for General Relativity. International Journal of Modern Physics D, 18, 2275-2282. https://arxiv.org/abs/0905.2502 https://doi.org/10.1142/S0218271809015904

[27] Clarkson, C. and Seahra, S. (2007) A Gravitational Wave Window on Extra Dimensions. Classical and Quantum Gravity, 24, F33. https://doi.org/10.1088/0264-9381/24/9/F01 


\section{Appendix A: Entropy Generation via Ng's Infinite Quantum Statistics}

The author brings up entropy development as given by [6], [9]. Furthermore, information counts, as discussed in this appendix tie in with information packing as brought up in the conclusion of the present paper. How do relic high frequency gravitational waves inter relate experimentally with the nucleation of short wave length relic gravitons? A small graviton creation volume, $V$, for relic gravitons of a high frequency ( short wave length) right after the big bang would be consistent Graviton volume $V$ for nucleation is tiny, well inside inflation. So the log factor drops out of entropy $S$ if $V$ is chosen properly for both Equation (C.1) and Equation (C.2). Ng's [9] result begins with a modification of the entropy/partition function $\mathrm{Ng}$ used in an approximation of temperature and its variation with respect to a spatial parameter, starting with temperature $T \approx R_{H}^{-1}$ ( $R_{H}$ can be thought of as a representation of the region of space of the particles in question). Furthermore, assume that the volume of space to be analyzed is of the form $V \approx R_{H}^{3}$ and look at a preliminary numerical factor we shall call $N \sim\left(R_{H} / l_{P}\right)^{2}$, where the denominator is Planck's length (on the order of $10^{-35}$ centimeters). We also specify a "wavelength" parameter $\lambda \approx T^{-1}$. So the value of $\lambda \approx T^{-1}$ and of $R_{H}$ are approximately of the same order of magnitude. Now this is how Jack $\mathrm{Ng}$ [9] changes conventional statistics: he outlines how to get $S \approx N$, which with additional arguments we refine to be $S \approx\langle n\rangle$ (where $\langle n\rangle$ is graviton density). Begin with

$$
Z_{N} \sim\left(\frac{1}{N !}\right) \cdot\left(\frac{V}{\lambda^{3}}\right)^{N}
$$

This, according to $\mathrm{Ng}$, leads to entropy of the limiting value of, if $S=\left(\log \left[Z_{N}\right]\right)$

$$
S \approx N \cdot\left(\log \left[V / N \lambda^{3}\right]+5 / 2\right) \underset{\text { Ng-infinite-Quantum-Statistics }}{\longrightarrow} N \cdot\left(\log \left[V / \lambda^{3}\right]+5 / 2\right) \approx N
$$

But $V \approx R_{H}^{3} \approx \lambda^{3}$, so unless $N$ in Equation (A.2) above is about 1, $S$ (entropy) would be $<0$, which is a contradiction. Now Equation (A.2) is where [9] introduces removing the $N$ ! term in Equation (A.1) above, removing the expression of $N$ inside the Log expression in Equation (A.2).

\section{Appendix B: Essential Gravitational Wave, and Experimental Gravity Constraints}

Keep in mind as well that there has been recent confirmation by Abbot [24] as to the existence of gravitational waves, which further extends what was brought up by Abbot, et al. of the LIGO observational team, which is in terms of black hole binaries, which further confirms the solutions of the issues, brought up by Abbot in [25] as well as also room to explore the insights brought up by Corda in [26] which await further investigation. In addition the speculation that extra dimensions [27] are involved becomes a testable datum, especially with the emerging science of gravitational wave astronomy. Which is of foundational import. 